Nach Veröffentlichtung der Schweizer UMTS-Studie (vgl. Kasten):

\title{
AefU bleiben bei Moratoriumsforderung für Mobilfunkanlagen
}

Dr. med. Bernhard Aufdereggen, Zentralvorstandsmitglied AefU

* Die Studie wurde in der Fach zeitschrift «Environmental health perspectives» veröffentlicht. Via Abstract (unter www.ehponline.org/docs/2006/ 8934/abstract.html) kann online kostenfrei auf den Volltext zugegriffen werden.

Korrespondenz:

Ärztinnen und Ärzte

für Umweltschutz

Postfach 111

CH-4013 Basel

Tel. 0613224949

info@aefu.ch

www.aefu.ch
Die Ärztinnen und Ärzte für Umweltschutz halten weiterhin an ihrer Forderung nach einem Moratorium im Weiterausbau der Mobilfunkinfrastruktur fest. Sie weisen darauf hin, dass sich ihre Haltung durch die Ergebnisse der Schweizer UMTS-Studie nicht verändert hat. Diese befasst sich nur mit Kurzzeiteffekten. Untersuchungen im Ausland weisen auf gesundheitlich negative Effekte der Langzeitbelastung durch Mobilfunkantennen hin. Beunruhigend sind die Veränderung genetischen Materials im Zellexperiment unter Einfluss von Hochfrequenzstrahlung und die offenen Fragen bezüglich Hirntumorrisiko und Handybenutzung. Vor dem Weiterausbau der Mobilfunkinfrastruktur sollen die Resultate des Nationalen Forschungsprogramms (NFP 57) abgewartet werden. In der Zwischenzeit fordern die Ärztinnen und Ärzte für Umweltschutz im Sinne der persönlichen Vorsorge alle Menschen auf, sich - aber insbesondere ihre Kinder - so wenig als möglich der Mobilfunkstrahlung auszusetzen.

\section{Experimentell nur kurzfristige Effekte untersucht}

Die holländische TNO-Studie hatte Effekte der kurzzeitigen UMTS-Exposition auf Wohlbefinden und kognitive Funktionen nachgewiesen. Die Schweizer UMTS-Studie zeigte diese Effekte nicht. Die Ergebnisse der TNO-Studie wurden nicht bestätigt.

Die Ärztinnen und Ärzte für Umweltschutz haben nach der Publikation der TNO-Studie ein Moratorium für UMTS-Antennen gefordert. Soll dieses Moratorium nun nicht mehr gelten? Wir halten weiterhin an einem Moratorium für den Weiterausbau der Mobilfunktechnologie fest, da einerseits die Resultate aktuell laufender Untersuchungen in Dänemark, Grossbritannien und Japan bis zur definitiven Beurteilung der Kurzzeitexposition abgewartet werden müssen. Andererseits werden mit der Versuchsanordnung, wie sie in der holländischen und der Schweizer Studie benutzt wurde, nur kurzfristige Effekte untersucht werden. Die Versuchspersonen wurden jeweils nur 45 Minuten dem Signal ausgesetzt. In der Realität können die Menschen dem
Signal einer Mobilfunkantenne während 24 Stunden ausgesetzt sein.

\section{Langzeiteffekte der Mobilfunkstrahlung befürchtet}

Langzeiteffekte über Monate und Jahre werden durch experimentelle Kurzzeituntersuchungen nicht erfasst. Mobilfunkantennen aber strahlen 24 Stunden am Tag. Eine erste aufsehenerregende Studie haben dieses Jahr Forscher der Universität Wien publiziert. Sie stellten fest, dass in der Umgebung von Mobilfunkantennen je nach Intensität der Strahlung mehr Menschen über unspezifische Symptome, wie Kopfschmerzen und Konzentrationsstörungen, klagten. Wir verlangen entsprechende Untersuchungen auch in der Schweiz, denn gemäss einer Befragung bei Schweizer Ärztinnen und Ärzten sind zwei Drittel der Befragten der Meinung, dass es Personen gibt, bei denen Gesundheitsbeschwerden durch elektromagnetische Felder ausgelöst werden. Schilderungen von elektrosensiblen Patientinnen und Patienten halten sie oft für plausibel. Fast die Hälfte der Ärztinnen und Ärzte ist der Meinung, dass die Behörden nicht genug unternehmen, um die Bevölkerung zu schützen.

Untersuchungen in verschiedenen Ländern zur Frage des Zusammenhanges zwischen Handybenutzung und Hirntumorrisiko haben widersprüchliche Resultate ergeben. In einzelnen Studien konnten Zusammenhänge insbesondere bei langjähriger Benutzung von Mobiltelefonen und Hirntumoren festgestellt werden.

Zur Vorsicht im Umgang mit Mobilfunk mahnen auch Ergebnisse einer Studie, die im Zell-

\section{Die Schweizer Mobilfunkstudie}

Ein Schweizer Forschungskonsortium hat keinen negativen Einfluss der Strahlung einer UMTS-Mobilfunkbasisstation auf das Wohlbefinden und die kognitiven Fähigkeiten nachweisen können*. Die Forschenden konnten damit die Befunde einer holländischen Studie aus dem Jahr 2003, die gesundheitliche Folgen der Mobilfunkstrahlung festgestellt hatte, nicht bestätigen. 
experiment Veränderungen des genetischen Materials unter Einfluss von hochfrequenter Strahlung nachgewiesen hat.

\section{Weitere Untersuchungen in der Schweiz geplant}

Der Bundesrat hat im letzten Jahr beschlossen, mit einem Nationalen Forschungsprogramm (NFP 57) die Auswirkungen nichtionisierender Strahlung auf den Körper untersuchen zu lassen. In Ergänzung zur internationalen Forschung untersuchen Schweizer Forscherinnen und Forscher in den nächsten vier Jahren die Effekte der nichtionisierenden Strahlung und damit auch des Mobilfunks. Diese Forschung ist in ihrer Unabhängigkeit höher einzustufen, da sie nicht mit Industriegeldern finanziert wird.

\section{Rolle der Mobilfunkanbieter}

Die Mobilfunkanbieter versuchen oft, die gesundheitlich negativen Effekte der Mobilfunkstrahlung herabzumindern und die Resultate kritischer Studien in einem negativen Licht dastehen zu lassen. Sie behelfen sich dabei entsprechender PR-Instrumente, wie dem Forum Mobil, und entsprechender Experten, die die Hinweise auf gesundheitlich negativen Einfluss der Mobilfunkstrahlung herabzumindern versuchen. Ein nicht kleiner Teil der Studien zu den Auswirkungen der Mobilfunkstrahlung auf die Gesundheit werden von den Anbietern direkt finanziert. Inwieweit die direkte Finanzierung solcher Studien deren Resultate beeinflusst, muss näher untersucht werden.

\section{Persönliche Vorsorge beim Handygebrauch}

Wir empfehlen die Massnahmen zur Reduktion der Strahlenbelastung beim Handytelefonieren, wie sie das Bundesamt für Gesundheit vom Hinweis auf strahlungsarme Handys bis zu «Keine Kinder ans Handy!» vorschlägt (bag.admin.ch/ themen/strahlung). 